\title{
Enhanced plane wave expansion analysis for the band structure of bulk modes in two-dimensional high-contrast solid-solid phononic crystals
}

\author{
Mohammadhosein Ghasemi Baboly ${ }^{1}$, Yasser Soliman ${ }^{2}$, Mehmet F. Su ${ }^{1,2}$, Charles M. \\ Reinke $^{3}$, Zayd C. Leseman ${ }^{1}$ and Ihab El-Kady ${ }^{1,2,3 *}$ \\ ${ }^{1}$ Mechanical Engineering Department, University of New Mexico, Albuquerque, \\ NM, USA \\ ${ }^{2}$ Electrical and Computer Engineering Department, University of New Mexico, \\ Albuquerque, NM, USA \\ ${ }^{3}$ Department of Applied Photonic Microsystems, Sandia National Laboratories, \\ Albuquerque, NM, USA
}

\begin{abstract}
Plane wave expansion analyses that use the inverse rule to obtain the Fourier coefficients of the elastic tensor instead of the more conventional Laurent's rule, exhibit faster convergence rates for solid-solid phononic crystals. In this work, the band structure convergence of calculations using the inverse rule is investigated and applied to the case of high acoustic impedance contrast solid-solid phononic crystals, previously known for convergence difficulties. Results are contrasted to those obtained with the conventional plane wave expansion method. The inverse rule is found to converge at a much rate for all ranges of impedance contrast, and the ratio between the computational times needed to obtain a convergent band structure for a high-contrast solid-solid phononic crystal with the conventional plane wave expansion method using 1369 reciprocal lattice vectors is as large as 6800:1. This ratio decreases for material sets with lower impedance contrast; however, the inverse rule is still faster for a given error threshold for even the lowest impedance contrast phononic crystals reported in the literature. This convergence enhancement is a major factor in reconsidering the plane wave expansion method as an important tool in obtaining propagating elastic modes in phononic crystals.
\end{abstract}




\section{INTRODUCTION}

Phononic crystals (PnCs) are the acoustic analogue of photonic crystals, in which periodically placed inclusions located in a homogenous host material scatter incident mechanical waves. This results in anomalous phonon dispersion for propagating modes, including the possibility of a range of frequencies that are completely reflected by the crystal, called phononic band gap (PnBG). The propagation of acoustic waves in PnCs has attracted a lot of interest due to their potential applications in acoustic imaging, filtering, and isolation ${ }^{1}$. The control of the frequency, bandwidth, and strength of the PnBG is very important for the functionality of such devices. In addition, maintaining a reasonable filling fraction or radius to lattice pitch ratio $(r / a)$, achieved by having a low mismatch in the velocity of wave propagation between the $\mathrm{PnC}$ material constituents, is essential for ease and repeatability of fabrication of high-frequency miniaturized $\mathrm{PnCs}^{1}$. However, the tradeoff is that the width of the band gap is mainly proportional to the impedance mismatch between the host and the inclusion materials ${ }^{2}$. A schematic of a PnC, along with the main parameters, is shown in Figure 1.

Many emerging applications of PnCs operate in the $\mathrm{MHz}$ and $\mathrm{GHz}$ frequency bands ${ }^{3-5}$. In these frequency regions, only PnCs based on solid-solid or solid-air (vacuum) material sets yield practical devices. Fluid-solid based PnCs, in spite of being the focus of much interest in macro-scale theoretical and experimental studies ${ }^{6,7}$, are impractical in the higher frequency ranges because of loss considerations and fabrication difficulties. Therefore most recent attention has been on PnCs with high impedance contrast solid- 
solid (HCSS) material sets wherein the acoustic impedance of the inclusion material $\left(\mathrm{Z}_{\text {inc }}\right)$ is greater than that of the matrix material $\left(Z_{\mathrm{cu}}\right)$, i.e. $Z_{\mathrm{inc}} / Z_{\mathrm{cu}}>1$.

One of the major drawbacks of the plane-wave expansion (PWE) method is convergence difficulties, especially when dealing with HCSS PnCs. The conventional fix is to use an ever-increasing number of reciprocal lattice vectors (RLVs), which ultimately translates

into large computational demands ${ }^{2,5-16}$. In this article, we show that using the inverse rule $^{13}$ to calculate the Fourier coefficients of the elastic tensor structure matrix allows for a very small number of RLVs to be sufficient for PWE convergence in determining the band structure of bulk acoustic waves for HCSS PnCs. A test system of tungsten (W) rods arranged in a square lattice in silica $\left(\mathrm{SiO}_{2}\right)$ is chosen for study, as this is a solid-solid material set with very high impedance contrast.

First an overview of the PWE method is presented, and then discussion on convergence issues with the conventional PWE (CPWE) and the new (i.e. inverse rule) PWE (NPWE) formulations ensues. Next, the PWE results are compared to FDTD and FEM simulations for two different material sets. Finally, the results are summarized and conclusions are drawn.

\section{THE PLANE-WAVE EXPANSION METHOD FORMULATION}

The PWE method is described in great detail in the literature ${ }^{17,18}$. To summarize, for a two-dimensional $\mathrm{PnC}$ we start with the equation of motion for the displacement vector 
$\vec{u}_{j}(\vec{r}, t)$ in an inhomogeneous linear elastic medium with no body force, which can be written as

$$
\rho(\vec{r}) \ddot{u}(\vec{r}, t)_{j}=\frac{\partial}{\partial x_{i}}\left(C_{i j k l}(\vec{r}) \frac{\partial u(\vec{r}, t)_{k}}{\partial x_{l}}\right)
$$

where $\vec{r}=\left(x_{1}, x_{2}, z\right)=(\vec{x}, z)$ is the position vector, and $\rho(\vec{r})$ and $C_{i j k l}(\vec{r})$ are the positiondependent mass density and elastic stiffness tensors, respectively.

Utilizing the Fourier transformation of the spatially-periodic inclusions, the material properties can be expanded, with respect to the two-dimensional RLVs, $\vec{G}=\left(G_{x}, G_{y}\right)$, in the Fourier series

$$
\begin{aligned}
& \rho(\vec{x})=\sum_{\vec{G}} e^{i \vec{G} \cdot \vec{x}}\left[\rho_{\vec{G}}\right] \text { and } \\
& C_{i j k l}(\vec{x})=\sum_{\vec{G}} e^{i \vec{G} \cdot \vec{x}}\left[C_{\vec{G}}^{i j k l}\right],
\end{aligned}
$$

where $\rho_{\vec{G}}$ and $C_{\vec{G}}^{i j k l}$ are the corresponding Fourier coefficients, defined as

$$
\begin{aligned}
& \rho_{\vec{G}}=A_{u c}^{-1} \int \rho(\vec{x}) e^{i \vec{G} \cdot \vec{x}} d^{2} \vec{x} \text {, and } \\
& C_{\vec{G}}^{i j k l}=A_{u c}^{-1} \int C_{i j k l}(\vec{x}) e^{i \vec{G} \cdot \vec{x}} d^{2} \vec{x},
\end{aligned}
$$


and $A_{u c}$ is the area of the primitive unit cell of a two-dimensional PnC, as described by Ref. ${ }^{19}$. Expanding the displacement vector utilizing Bloch's theorem ${ }^{20}$ in a Fourier series, we get

$$
u_{j}(\vec{r}, t)=\sum_{\vec{G}} e^{i(\vec{k}+\vec{G}) \cdot \vec{x}-i \omega t}\left(e^{i k_{z} z} \vec{A}_{\vec{G}}^{j}\right)
$$

where $\vec{k}=\left(k_{x}, k_{y}\right)$ is the Bloch wave vector, $\omega$ is the angular frequency, $k_{z}$ is the wave number along the $z$-direction, and $\vec{A}_{\vec{G}}^{j}$ is the amplitude of the displacement vector. As $k_{z}$ approaches zero, Eqn. (1) degenerates into the displacement vector of bulk acoustic waves. Substituting Eqns. (2, $3 \& 6$ ) into (1), and after collecting terms and setting $k_{z}$ to zero, a generalized eigenvalue problem is formed:

$\mathbf{C} \cdot \mathbf{U}=\mathbf{0}$,

where

$$
\begin{aligned}
& \mathbf{C}=\left[\begin{array}{ccc}
M 1 & L 1 & U 1 \\
L 2 & M 2 & U 2 \\
W 1 & W 2 & M 3
\end{array}\right] \text {, and } \\
& \mathbf{U}=\left[\begin{array}{c}
A_{\vec{G}}^{1} \\
A_{\vec{G}}^{2} \\
A_{\vec{G}}^{3}
\end{array}\right] .
\end{aligned}
$$


Matrix elements, $M 1, \underline{L} 1$, etc., are each of size $n \times n$, forming a C matrix of size $3 n \times 3 n$, where $n$ is the total number of RLVs used in the Fourier expansion. The components of matrix $C$ depend only on $\vec{k}, \vec{G}, \omega$, and the material properties $\rho_{\vec{G}}$ and $C_{\vec{G}}^{i j k l}$. The $(\omega, k)$ pairs satisfying Eqn. (7) define the dispersion relations of bulk acoustic waves propagating in the two-dimensional PnC.

For materials with orthorhombic symmetry or higher, the components U1, U2, W1 and W2 in matrix $\mathrm{C}$ are zero and Eqn. (7) can be decoupled into two different polarizations as follows:

$$
\left[\begin{array}{cc}
M 1 & L 1 \\
L 2 & M 2
\end{array}\right]\left[\begin{array}{l}
A_{\vec{G}}^{1} \\
A_{\vec{G}}^{2}
\end{array}\right]=0
$$

for mixed polarization modes (longitudinal and shear), and

$$
[M 3]\left[A_{\vec{G}}^{3}\right]=0
$$

for modes with polarization displacement along the axial direction of the cylinders, the $z-$ direction shown in Fig. 1. In all PWE calculations in this paper, only the mixed polarizations will be calculated, as given by Eqn. (10). 
The formulation above has been known to have convergence issues, particularly when considering HCSS materials ${ }^{2,5-16}$. To enhance the convergence, it is argued that one should use an alternative formulation using the inverse rule when obtaining the Fourier coefficients of $C_{i j k l}(\vec{x})$ instead of the conventionally used Laurent's rule, according to the procedure of Fourier factorization of a product of two piecewise smooth, bounded, periodic functions. This leads to substituting $\left[C_{i j k l}\right]$ with $\left[\frac{1}{C_{i j k l}}\right]^{-1}$ in Eqn. (3) above ${ }^{21}$, where the brackets denote the Toeplitz matrix of the enclosed function. The inverse rule was observed to yield a faster and more stable algorithm, especially in the case of HCSS PnCs, in agreement with the suggestion and observations inRef. ${ }^{10}$. The dispersion relations obtained using this "new" PWE (NPWE) method are presented and discussed in the subsequent sections.

\section{CONVERGENCE COMPARISON FOR HCSS PnC}

In this section, the number of RLVs needed for the convergence of the band structure modes for HCSS PnCs is studied. PnCs comprised of cylindrical W inclusions arrayed in a square lattice arrangement in $\mathrm{SiO}_{2}$ background material were chosen. This solid-solid material set has one of the largest impedance mismatches between solids commonly used in the microelectronics fabrication industry, as shown in Table 1.

Using Eqn. (10) above, the dispersion relations are calculated for bulk acoustic waves in a $\mathrm{W} / \mathrm{SiO}_{2}$ PnC using the NPWE method to compare with dispersion relations obtained with the "conventional" PWE (CPWE) method that uses Laurent's rule. Fig. 2 shows a 
comparison between the band structure of bulk acoustic waves for the $\mathrm{W} / \mathrm{SiO}_{2} \mathrm{PnC}$ using the NPWE with 25 RLVs vs. the CPWE with 81 and 1369 RLVs.

Baseline values for comparison are gathered from the finite element method (FEM), which is one of the most efficient methods used in various areas of science and technology for solving many partial differential equations and integral equations over complicated domains. In general FEM is more accurate and can be applied to a larger class of solid materials compared to the other methods for solving elastic wave propagation equations and is generally used in the literature to output reliable results ${ }^{22-24}$.

The first five modes are chosen for comparison because the band gap for this structure lies between the third and the fourth bands, and the band gap width is arguably the most important characteristic relating to the functionality of a PnC. Therefore, predicting the band gap width and location is the most important piece of information to be supplied with a modeling technique such as this for PnCs. $\mathrm{s}$

It is clear from Fig. 2 that using NPWE enhances convergence of the modes dramatically. The superiority of the new formulation is evident when considering the band structure obtained using CPWE with 1369 RLVs as compared to NPWE using only 25 RLVs. The ratio of the computation time needed to obtain the band structure of a HCSS PnC for the CPWE using 1369 RLVs to that obtained using the NPWE with 25 RLVs is on the order of 6800:1, which is substantial. 
Convergence is further verified through comparison of the PnBG width with results from the finite-difference time-domain method (FDTD), which has been previously developed and verified ${ }^{25}$. Figure 3 shows a comparison of the relative bandgap width vs. $r / a$ (radius of an inclusion / spacing of the inclusions; see Fig. 1) for FDTD (red diamonds) and NPWE with 25 RLVs (green triangles). Variation of less than 5\% exists for $r / a$ values between 0.15 and 0.4 ; nearly all published HCSS PnCs were designed within this range. Figures 2 and 4 were created using $r / a=0.32$.

A comparison among the frequencies of the first five modes at the $\mathrm{X}$ point $\left(k=\frac{\pi}{a}(1,0)\right)$ is shown in Fig. 4. Percent error is reported versus the number of RLVs where the percent error is calculated against the band frequencies provided by the next finer resolution. For example, the percent error for 9 RLVs is compared against $25 \mathrm{RLVs}$, for 25 the baseline is results from 81, etc. $0 \%$ error means that the results are converged. It is clear from Fig. 4 that even with 1369 RLVs, modes obtained by CPWE are not fully converged, while only 49 RLVs are needed for the modes obtained by the NPWE method to give solutions within $0.5 \%$ error from those results obtained with 841 \& 1369 RLVs using NPWE. Although CPWE offers around 4\% error using 81 RLVs, $4 \%$ error of each of the bands bounding the PnBG would cause a significant error in the gap to mid-gap ratio, a normalized measure of the gap width, which is usually on the order of $25 \%$ at higher frequencies ${ }^{26}$. NPWE provides a remarkable convergence speed increase and gives results within $0.5 \%$ error in a fraction (around 0.00014 ) of the time needed by the CPWE to offer results within $1 \%$ error using 1369 RLVs. Also notice the pseudo-convergence CPWE shows at a lower number of RLVs (up to 81 RLVs), which can be deceiving, after 
which the modes start converging toward the values achieved by the NPWE with only 25 RLVs.

The band diagrams of Solid-solid PnCs made of five different pairs of materials found in the literature ${ }^{16,27-30}$ were also calculated using both methods with same number of RLVs. The RMS error for each method is calculated based on the FEM results as the reference. Figure 5 shows the normalized error, defined as the ratio of CPWE error to NPWE error, of each pair. This demonstrates that for all of the practical values of the impedance mismatch for commonly studied HCSS PnCs, NPWE converges better.

To study the accuracy of each method for different values of impedance mismatch, the elastic modulus of the inclusion was artificially varied from $10 \mathrm{GPa}$ to $2500 \mathrm{GPa}$ to cover the range that has been published in literature to date. Figure 6 indicates while CPWE is trustable for some ranges of the impedance mismatch, for $\mathrm{Z}_{\mathrm{inc}} / \mathrm{Z}_{\mathrm{cu}}$ values greater than 5 the normalized error starts to increase. For HCSS materials, the error for the CPWE method increases by over a factor of 5 (10 fold) more than for NPWE.

The above section showed the strong influence of the inappropriate use of the Laurent's rule to calculate the elastic tensor Fourier coefficients of the PWE technique ${ }^{10}$, and suggests that faster and more consistent convergence can be achieved with the new formulation. It also suggests that the lack of convergence of the PWE method should not be attributed to an inherent slow convergence of the Fourier series for the elastic parameters at the interfaces of the various materials comprising a PnC. 
It is worth noting that one could use a brute force numerical technique to solve the eigenvalue problem in Eqn. (7) by sampling over both $\omega$ and $\vec{k}$ and finding pairs satisfying Eqn. (7) ${ }^{8}$. However, although similar results are achieved by both methods, directly solving the eigenvalue problem is much faster than using a brute force sampling technique to obtain the band structure, even when shared memory multi-processing (such as OpenMP) is utilized, since the direct solution approach allows for sampling over one parameter only, namely $\vec{k}$. The discussion above regarding CPWE vs. NPWE applies to the brute force approach equally well, since identical eigenfrequencies are obtained whether the brute force sampling or the direct solution approach is undertaken.

\section{CONCLUSIONS}

The convergence behavior a new formulation of the plane wave expansion method for modeling bulk acoustic wave propagation in two-dimensional PnCs was investigated. It was shown that the band structure of high-contrast solid-solid PnCs, which was previously known for convergence difficulties, could be efficiently calculated as compared to the results obtained with the conventional plane wave expansion method. The new formulation is found to converge at a much faster rate, and the ratio between the computation time needed to obtain the converged band structure of a high-contrast solidsolid PnC for the conventional method using 1369 reciprocal lattice vectors vs. the new formulation using 81 vectors can be as high as around 6800:1, in high contrast solid-solid material sets. This convergence enhancement may prove to be a significant development in PnC modeling. 
* Author to whom correspondence should be addressed: ielkady@sandia.gov

\section{ACKNOWLDGMENTS}

The National Science Foundation, Division of CMMI under Award 1056077, supported this work. Sandia National Laboratories is a multi-program laboratory managed and operated by Sandia Corporation, a wholly owned subsidiary of Lockheed Martin Corporation, for the U.S. Department of Energy's National Nuclear Security Administration under contract DE-AC04-94AL85000.

1. Olsson III, R. H. \& El-Kady, I. Microfabricated phononic crystal devices and applications. Meas. Sci. Technol. 20, 012002 (2009).

2. Kee, C.-S., Kim, J.-E., Park, H. Y., Chang, K. J. \& Lim, H. Essential role of impedance in the formation of acoustic band gaps. J. Appl. Phys. 87, 1593 (2000).

3. Olsson, R. H., Fleming, J. G., El-kady, I. F., Tuck, M. R. \& Frederick, B. Tungsten Scatterer r AlN Coupler Release Holes Release Pit. 317-321 (2007).

4. Olsson, R. H., El-Kady, I. F., Su, M. F., Tuck, M. R. \& Fleming, J. G. Microfabricated VHF acoustic crystals and waveguides. Sensors Actuators A Phys. 145-146, 87-93 (2008).

5. El-Kady, I., Olsson, R. H. \& Fleming, J. G. Phononic band-gap crystals for radio frequency communications. Appl. Phys. Lett. 92, 233504 (2008).

6. Yang, S. et al. Focusing of Sound in a 3D Phononic Crystal. Phys. Rev. Lett. 93, 024301 (2004).

7. Khelif, A., Choujaa, A., Benchabane, S., Djafari-Rouhani, B. \& Laude, V. Guiding and bending of acoustic waves in highly confined phononic crystal waveguides. Appl. Phys. Lett. 84, 4400 (2004).

8. Vasseur, J., Deymier, P., Djafari-Rouhani, B., Pennec, Y. \& Hladky-Hennion, a-C. Absolute forbidden bands and waveguiding in two-dimensional phononic crystal plates. Phys. Rev. B 77, 085415 (2008).

9. Hsu, J.-C. \& Wu, T.-T. Efficient formulation for band-structure calculations of two-dimensional phononic-crystal plates. Phys. Rev. B 74, 144303 (2006). 
10. Cao, Y., Hou, Z. \& Liu, Y. Convergence problem of plane-wave expansion method for phononic crystals. Phys. Lett. A 327, 247-253 (2004).

11. Goffaux, C. \& Sánchez-Dehesa, J. Two-dimensional phononic crystals studied using a variational method: Application to lattices of locally resonant materials. Phys. Rev. B 67, 144301 (2003).

12. J. O. Vasseur, B. Djafari-Rouhani, L. Dobrzynski, M. S. Kushwaha, and P. H. Complete acoustic band gaps i n periodic fibre reinforced composite materials : the carbodepoxy composite and some metallic systems. Phys.: Condes. Matter 87598770 (1994).

13. Sigalas, M. M. \& Economou, E. N. Band structure of elastic waves in two dimensional systems. Solid State Commun 86, 141 (1993).

14. Sliwa, I. \& Krawczyk, M. Phononic Band Gap Width Control through Structural and Material Parameters in Two-Dimensional Phononic Crystals. 108, (2005).

15. J. O. Vasseur, B. Djafari-Rouhani, L. Dobrzynski \& P. A. Deymier. Acoustic band gaps in fibre composite materials of boron nitride structure. J. Phys. Condens. Matter 9, 7327 (1997).

16. Charles, C., Bonello, B. \& Ganot, F. Propagation of guided elastic waves in 2D phononic crystals. Ultrasonics 44 Suppl 1, e1209-13 (2006).

17. Tanaka, Y. \& Tamura, S. Surface acoustic waves in two-dimensional periodic elastic structures. Phys. Rev. B 58, 7958-7965 (1998).

18. Wu, T.-T., Huang, Z.-G. \& Lin, S. Surface and bulk acoustic waves in twodimensional phononic crystal consisting of materials with general anisotropy. Phys. Rev. B 69, 094301 (2004).

19. M. S. Kushwaha. 20.pdf. Recent Res. Devel. Appl. Phys 2, 743 (1999).

20. Kittel, C. Introduction to solid state physics. (Wiley, 1953).

21. Li, L. Use of Fourier series in the analysis of discontinuous periodic structures. $J$. Opt. Soc. Am. A 13, 1870-1876 (1996).

22. Djafari-Rouhani, B. et al. Absolute band gaps and waveguiding in free standing and supported phononic crystal slabs. Photonics Nanostructures - Fundam. Appl. 6, 32-37 (2008).

23. Veres, I. a. \& Berer, T. Complexity of band structures: Semi-analytical finite element analysis of one-dimensional surface phononic crystals. Phys. Rev. B 86, 104304 (2012). 
24. Zen, N., Puurtinen, T. a., Isotalo, T. J., Chaudhuri, S. \& Maasilta, I. J. Engineering thermal conductance using a two-dimensional phononic crystal. Nat. Commun. 5, 1-9 (2014).

25. Su, M. F., Olsson, R. H., Leseman, Z. C. \& El-Kady, I. Realization of a phononic crystal operating at gigahertz frequencies. Appl. Phys. Lett. 96, 053111 (2010).

26. Mohammadi, S., Eftekhar, A. A., Hunt, W. D. \& Adibi, A. High-Q micromechanical resonators in a two-dimensional phononic crystal slab. Appl. Phys. Lett. 94, 051906 (2009).

27. Reinke, C. M., Su, M., Ziaei-moayyed, M., Iii, R. H. O. \& Technique, A. P. E. Investigation of Full Bandgaps in Silicon Phononic Crystal Membranes with Tungsten and Air Inclusions. 519-522 (2010).

28. Soliman, Y. M. et al. Effects of release holes on microscale solid-solid phononic crystals. Appl. Phys. Lett. 97, 081907 (2010).

29. Lin, S.-C., Huang, T., Sun, J.-H. \& Wu, T.-T. Gradient-index phononic crystals. Phys. Rev. B 79, 094302 (2009).

30. Hsu, J.-C. \& Wu, T.-T. Efficient formulation for band-structure calculations of two-dimensional phononic-crystal plates. Phys. Rev. B 74, 144303 (2006).

Table 1 . Mass density $\rho$, longitudinal sound velocity $\mathrm{C}_{l}$, acoustic impedance $Z$, Young's modulus E, and Poisson's ratio $v$ for $\mathrm{W}$ and $\mathrm{SiO}_{2}$

\begin{tabular}{|c|c|c|c|c|c|}
\hline Material & $\rho\left(\mathrm{kg} / \mathrm{m}^{3}\right)$ & $\mathrm{C}_{l}(\mathrm{~m} / \mathrm{s})$ & $Z\left(\mathrm{Kg} /\left(\mathrm{m}^{2} \mathrm{~s}\right)\right.$ & $\mathrm{E}(\mathrm{GPa})$ & $v$ (unitless) \\
\hline Tungsten $(\mathrm{W})$ & 19300 & 4611 & 89 & 410 & .28 \\
\hline Silica $\left(\mathrm{SiO}_{2}\right)$ & 2200 & 5840 & 12.8 & 75 & .17 \\
\hline
\end{tabular}

Figure 1 - A demonstration of a phononic crystal showing the radius of the inclusions $r$ and the lattice pitch $a$, as well as the directions referred to in the derivation of the plane wave expansion method

Figure 2 - A comparison between the elastic band structure for the bulk $2 \mathrm{D}$ PnC made of an array of $W$ of cylindrical cross section, arranged in a simple cubic lattice \& embedded in a $\mathrm{SiO}_{2}$ background with an r/a ratio of 0.32 using the CPWE and NPWE formulations mentioned above. A dimensionless frequency, $\omega a / C_{t} i$ reported on the vertical axis, where $C_{t}$ is the transverse acoustic velocity in $\mathrm{SiO}_{2}$. Note the faster convergence of the NPWE with as low as 25 RLVs when compared to the conventional method for 81 RLVs and even 1369 RLVs. 
Figure 3 - Normalized band gap width $\left(\omega a / C_{t}\right)$ comparison vs. r/a ratio using FDTD (red diamonds) and the NPWE (green triangles) with only 25 RLVs showing the agreement in bandgap widths when both methods are utilized.

Figure 4 - The percent error in the first five bands at the $X$ point $\left(k=\frac{\pi}{a}(1,0)\right)$ showing the results obtained from using the CPWE and NPWE methods with 9, 25, 49, 81, 441, 841 and $1369 \mathrm{RLVs}$ for the $\mathrm{W} / \mathrm{SiO}_{2} \mathrm{PnC}$. Notice that only $49 \mathrm{RLVs}$ are needed for the modes obtained with the NPWE method to have an error less than $0.5 \%$. Also notice the pseudo-convergence the CPWE shows at a lower number of RLVs (up to 81 RLVs), which can be deceiving, after which the modes start converging.

Figure 5 -Normalized RMS Error calculated for practical materials used for PnCs for 961 RLVs. The error of each method is referenced against FEM. Normalized error is the ratio of CPWE error over NPWE error.

Figure 6-Normalized RMS Error calculated for different impedance mismatch. Impedance of inclusion is varied by changing the elastic modulus of it while keeping the impedance of the host material constant.

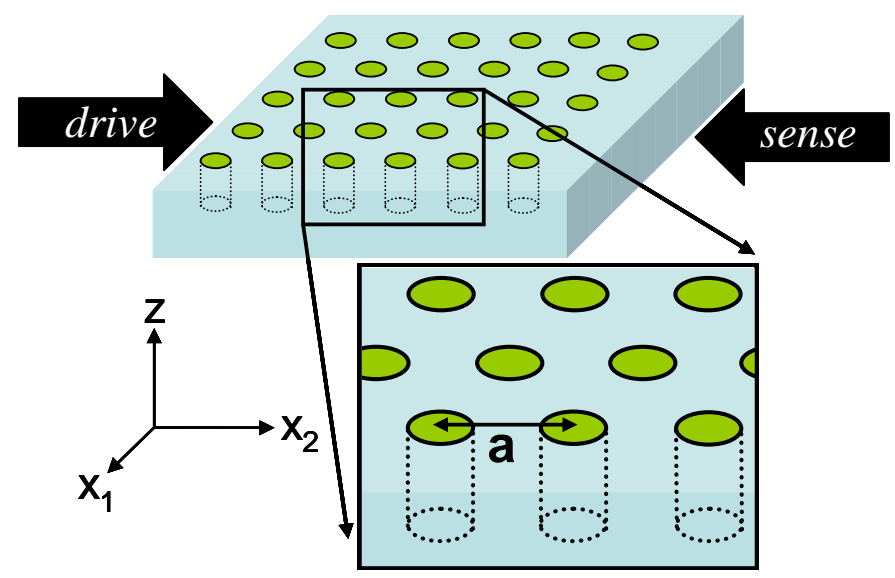

Figure 3 


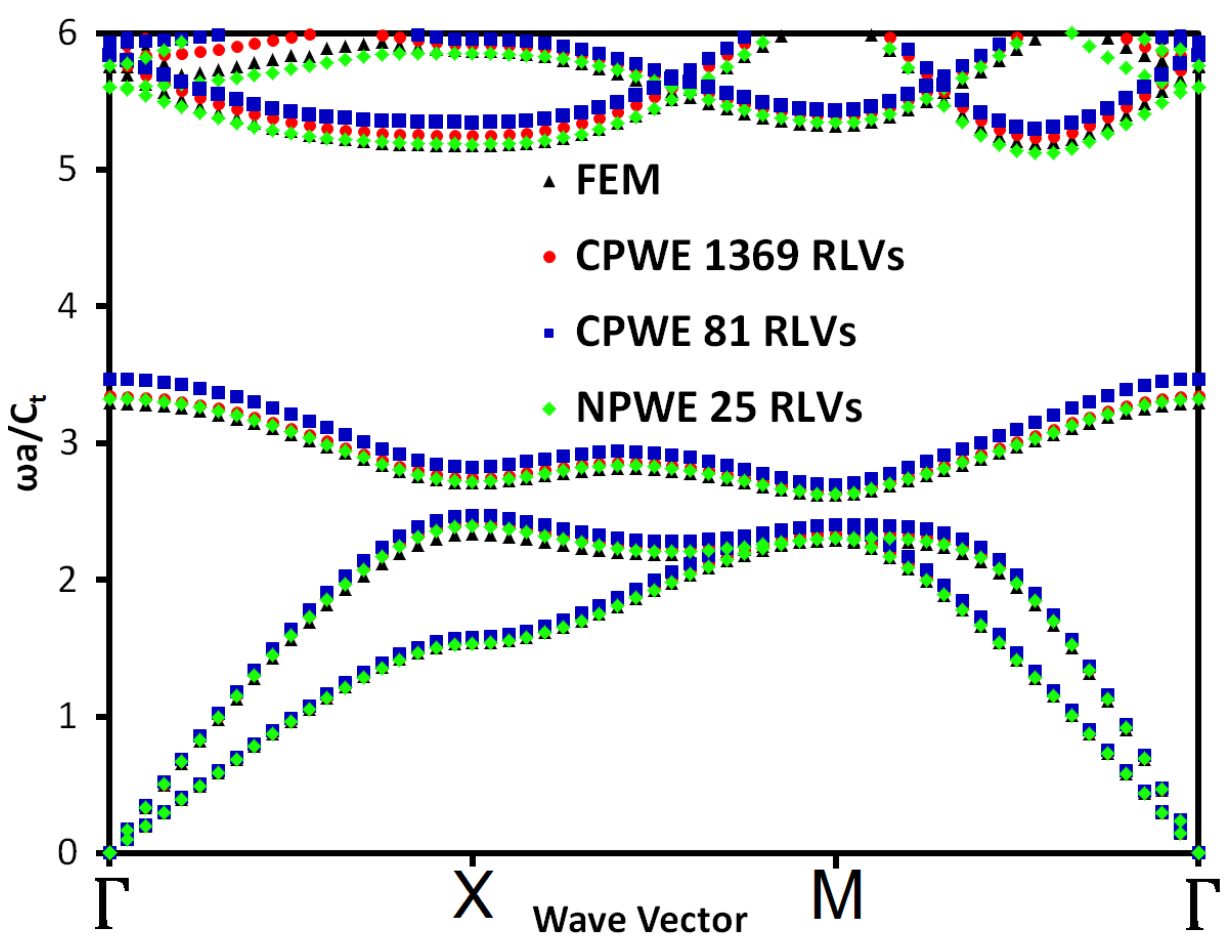

Figure 2

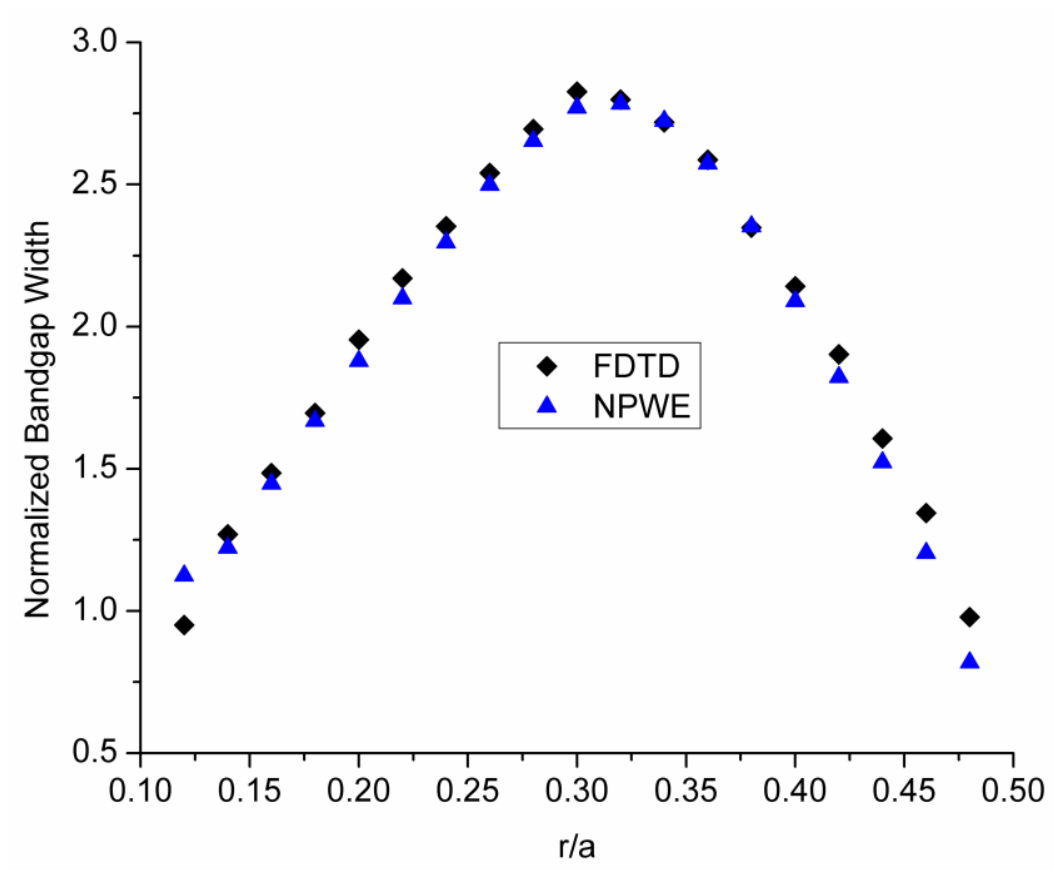

Figure 3 


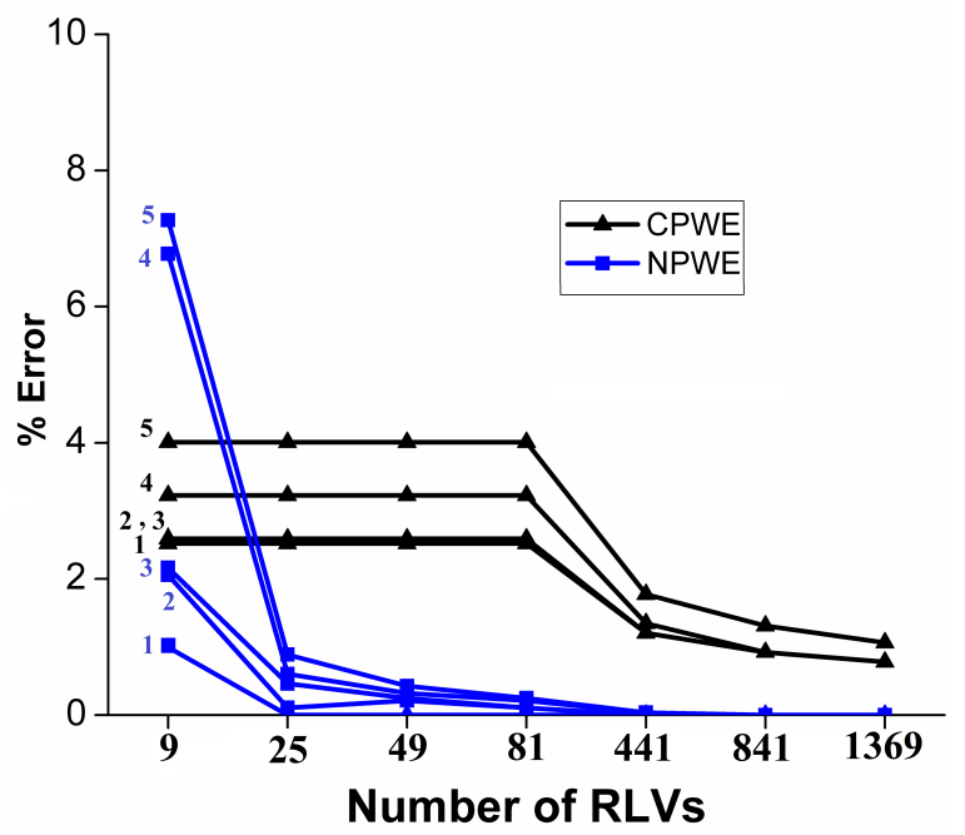

Figure 4

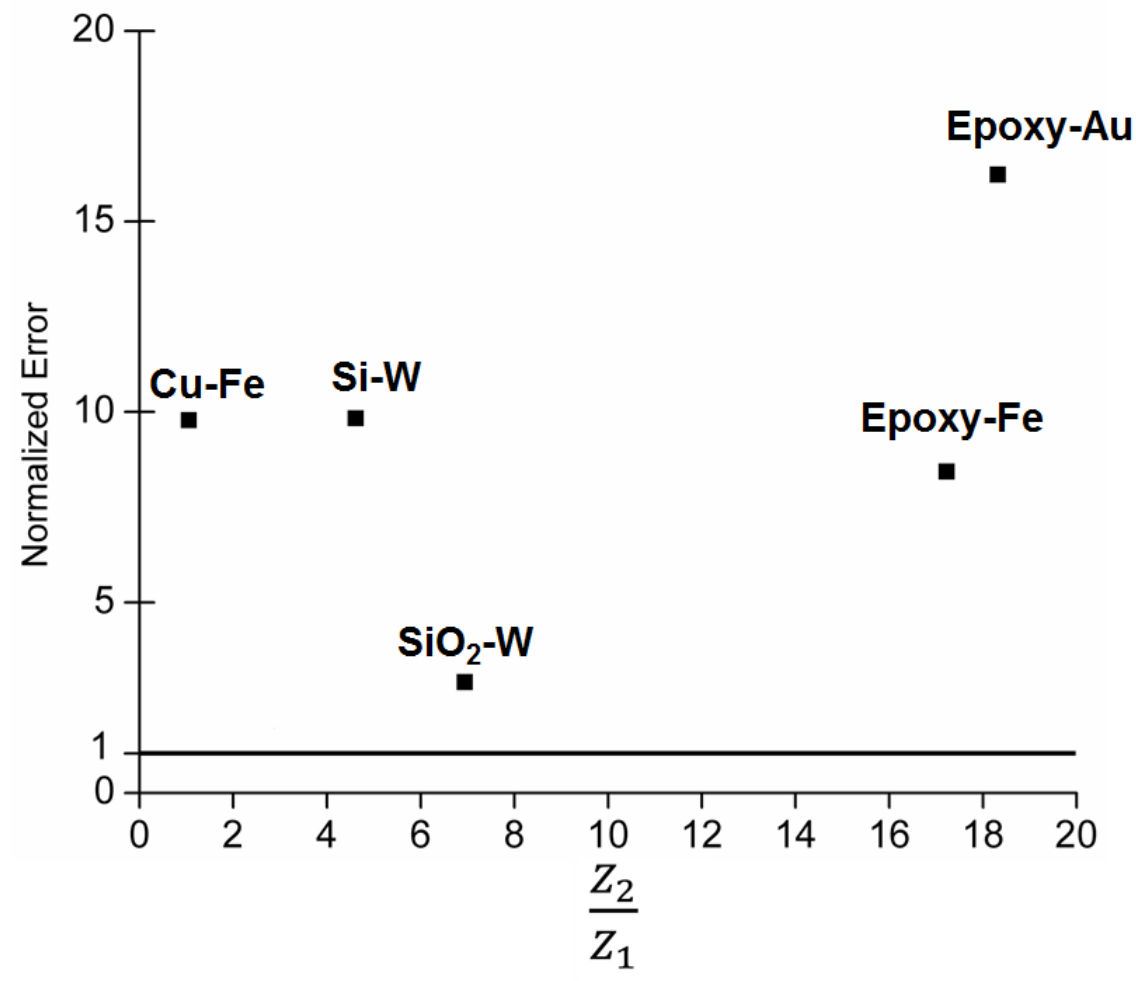

Figure 5 


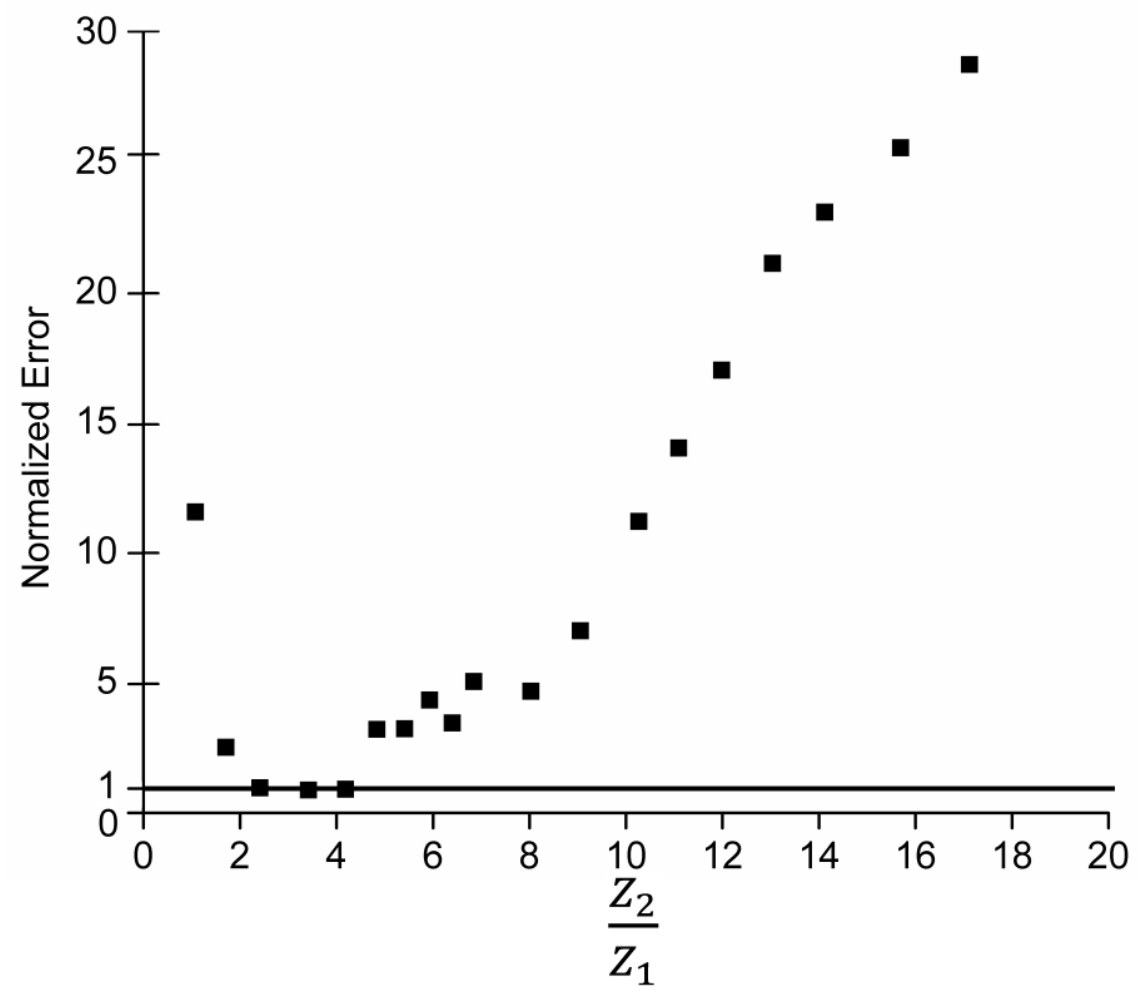

Figure 6 